(C) 2015 IEEE. Personal use of this material is permitted. Permission from IEEE must be obtained for all other uses, in any current or future media, including reprinting/republishing this material for advertising or promotional purposes, creating new collective works, for resale or redistribution to servers or lists, or reuse of any copyrighted component of this work in other works. 


\title{
A Measurement System for Radiated Transient Electromagnetic Interference Based on General Purpose Instruments
}

\author{
Marco A. Azpúrua, Marc Pous and Ferran Silva \\ Grup de Compatibilitat Electromagnètica (GCEM), Departament d'Enginyeria Electrònica (DEE) \\ Universitat Politècnica de Catalunya (UPC) \\ Barcelona, Spain \\ Email: marco.azpurua@upc.edu
}

\begin{abstract}
This paper presents a measurement system intended to be used to assets the radiated electromagnetic interference (EMI) in both time and frequency domains. In order to keep the measurement setup as accessible and practical as possible, the direct measurements are recorded with a general purpose digital oscilloscope and processed with a commonly available personal computer. The measurement system was validated for radiated emissions testing using well-known, controlled transient and continuous signals emulating typical interferences. The results are in satisfactory agreement with those provided by a conventional EMI receiver for different types of detectors. The proposed approach shows that, currently, it is possible to implement a timesaving, accurate and generally inexpensive time domain measurement system for radiated emissions that is capable to overcome the limitations of the superheterodyne EMI receivers regarding the measurement of discontinuous electromagnetic disturbances and also able to provide additional enhanced features to evaluate and troubleshoot EMI problems.
\end{abstract}

Keywords-Time domain measurements, electromagnetic interference, radiated emissions, spectral estimation, electromagnetic compatibility.

\section{INTRODUCTION}

Nowadays, an important cause of electromagnetic incompatibilities and interferences are broadband impulsive noise disturbances unintentionally produced, i.e., by sparks or switching power supplies that propagate as radiated signals affecting digital communication systems receivers as in-band interferences [1]. This is the case of several contemporary applications such as Digital Video Broadcasting Terrestrial (DVB-T), Trans European Trunked RAdio (TETRA), Radio Frequency IDentification (RFID) and GSM-Railway system that are susceptible of degradation in their performance under the impact of transient broadband interfering noise [2][3].

However, most of the standardized methods and measurement setups used for evaluating the radiated electromagnetic emissions were developed to protect analogue communication systems from interferences and, therefore, the aforementioned standards and their associated measuring equipment are not generally suitable to perform a proper assessment of the impact of radiated interference in such digital communication systems and applications [3]. Additionally, in general terms, the standard methods for evaluating the radiated electromagnetic emissions have evolved at a slower pace with respect to the communications technologies making them inadequate or insufficient to ensure electromagnetic compatibility (EMC) in situations as those described previously [4].

Consequently and in view of the limitations of the superheterodyne EMI receivers, important advances have been achieved in order to implement Time Domain Electromagnetic Interference Measurement (TDEMI) systems fully compliant with CISPR 16-1-1 standard. Those CISPR compliant FFTbased EMI receivers tend to reduce the measurement time spent in the conducted and radiated emissions test by several orders of magnitude [1][5]. Nevertheless, in general terms, those TDEMI systems have been more focused on emulating the functioning, specifications and performance of the stateof-the-art EMI receivers that operate in the frequency domain than in performing a more comprehensive evaluation of the electromagnetic disturbance in the time domain [6].

Perhaps, the approach taken so far in the TDEMI measurement systems propitiated their recognition as (FFT-based) test instruments for standard-compliant measurements according to the International Special Committee on Radio Interference (CISPR) requirements, specifically by means of the publication of the Amendment 1 to the third edition of CISPR 16-1-1 [7]. Notwithstanding, the inclusion of the TDEMI measurement systems in the CISPR 16-1-1 standard neither attempt to address the study of electromagnetic disturbances in the time domain nor makes the test equipment less expensive. In fact, the large majority of the commercially available EMI receivers with the optional time domain scan functionality are a very sophisticated piece of hardware that is neither affordable for small laboratories nor is suitable for in-situ measurements [8].

Aware of the increasingly importance of evaluating the impact of transient, discontinuous and intermittent EMI while developing the means for making the maximum of commonly available laboratory equipment, this work is intended to introduce a low-cost time domain EMI measurement system for radiated emissions, that uses as general purpose digital oscilloscope to measure and acquire the signals that are later processed to obtain not only the estimated spectral content of the EMI, but also useful information on the time domain characteristics of the electromagnetic disturbance.

In the following sections, the aforementioned TDEMI system will be explained and the results from its experimental validation will be provided and compared with those obtained using a conventional frequency domain EMI receiver. 


\section{OVERVIEW OF TDEMI MEASUREMENT SySTEMS}

In general terms, a TDEMI measurement system can be described by the block diagram shown in Figure 1[9]. Those TDEMI systems can be employed, in theory, for the measurement of either the radiated EMI or conducted EMI. For the measurement of radiated electromagnetic interference a broad-band antenna shall be used, while for the measurement of conducted EMI corresponds either a current clamp or line impedance stabilization network. Sometimes the measured signal passes through a preamplifier and then it is low-pass filtered for band limiting proposes, so the signal fulfills the Nyquist condition for the maximum sampling rate supported by the TDEMI system. Then, in the analog-to-digital converter (ADC), the signal is digitized and then it is stored. Finally, the amplitude spectrum is computed via a spectral estimation techniques.

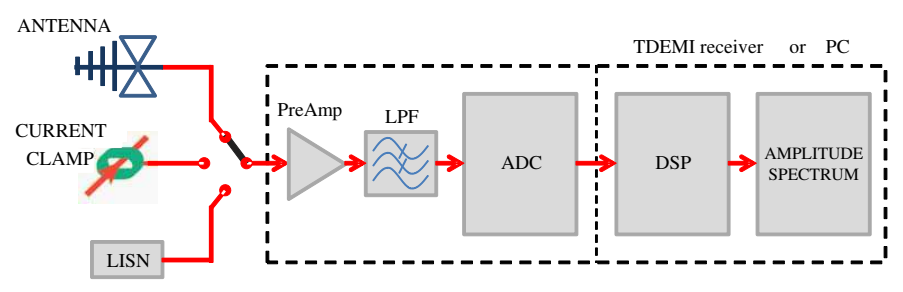

Fig. 1. Block diagram of a Time-Domain EMI measurement system.

On the other hand, from the standpoint of the signal acquisition and processing stage, a TDEMI system is more naturally described by the flow chart shown in Figure 2.

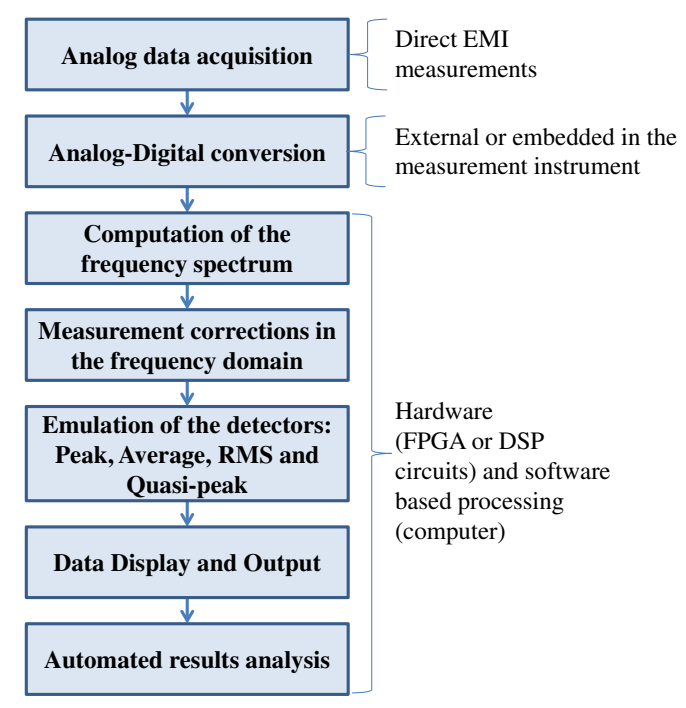

Fig. 2. General flow chart of the TDEMI measurement processing.

In that sense, the data acquisition process for a TDEMI measurement system begins when the analog signal is sampled either directly by the measuring instrument (i.e., digital oscilloscope) or externally by means of ADC or digitizers. Then, the EMI data are transferred from the main memory to the device that will process it. For the commercial FFTbased EMI receivers this process is performed internally by the built-in processors and software [6]. However, a customized TDEMI measurement system may carry on the digital signal processing with the aid of specific software running on a personal computer. Then, the data are transferred to a personal computer via some communication port, such as the General Purpose Interface Bus (GPIB). Subsequently, the amplitude spectrum is digitally computed using the FFT (or some variant of), periodograms or other spectral estimation methods [10]. Later, the errors due to the frequency dependence of the antenna factor, the attenuation in the transmission line, the gain of the preamplifier, the frequency response of the anti-aliasing filter are corrected. Next, a mathematic emulation of the peak, RMS, average and quasi-peak detectors is made, either by applying a correction factor dependent on the pulse repetition frequency [11] or by means of the application of a digital infinite impulse response filter [12]. Additionally, other signal processing techniques can be used in order to reduce the impact of the noise but this has varied upon specific implementations. Finally, the results must be displayed and compared with the respective limit lines to provide a judgment about the test result regarding the EMI in the measured frequency band. For studying non-stationary signals, a spectrogram representation of EMI has also been used previously[13].

\section{GenERAL DESCRIPTION OF THE TDEMI MEASUREMENT SYSTEM}

\section{A. Hardware}

Even if the measurement method is, by itself, neither restricted in terms of the maximum measurable frequency, dynamic range nor noise floor, it is important to indicate the overall technical specification of the hardware in order to emphasize the use of general purpose instruments as a key aspect of our approach. In that sense, the developed TDEMI measurement system uses a general purpose digital oscilloscope and a personal computer for pre- and post-processing the results in both the time an the frequency domain. Specifically, the make and model of the oscilloscope used is Tektronix DPO 7104, which has a $1 \mathrm{GHz}$ bandwidth, a maximum sampling rate of $20 \mathrm{GSamples} / \mathrm{s}$, and up to 8 bit ( $>11$ bit with Hi-Res mode) ADC resolution (6.7 effective number of bits). Thus, the maximum frequency measurable by the oscilloscope is limited to $1 \mathrm{GHz}$ by the hardware, making it unnecessary to apply an additional low-pass filter as shown in Figure 1. The maximum theoretical dynamic range for a harmonic input signal with an amplitude equal to the ADC full-scale input is $110 \mathrm{~dB}(74$ $\mathrm{dB}$ plus the processing gain and at full range signal), while typically the effective dynamic range is about $78 \mathrm{~dB}$.

\section{B. Sampling scheme}

It is well-known that, according to the (uniform) sampling theorem, the minimum required sampling rate must be at least twice the upper frequency limit of the measured band in order to be able to reconstruct the sampled signal, that is 2 GSamples/s for a TDEMI system intended to measure up to $1 \mathrm{GHz}$. However, in order to avoid the aliasing influence, the generally recommended criteria is to select a sampling frequency, $f_{s}$, of approximately four times the upper frequency bound of the spectrum to be measured, that is, $4 f_{\max }$ [14]. This particular TDEMI measurement system implementation selects, from a set of possible sampling frequencies configurable in the oscilloscope, the immediately sampling rate higher than 
$4 f_{\text {max }}$. For example, considering a measure between $30 \mathrm{MHz}-$ $1 \mathrm{GHz}, f_{s}$ is set to $5 \mathrm{GSamples} / \mathrm{s}$ since $f_{\max }=1 \mathrm{GHz}$. On the other hand, if measuring in the band $100 \mathrm{MHz}-300 \mathrm{MHz}$, $f_{s}$ is set to 1.25 GSamples/s. The main objective in adjusting $f_{s}$ is to use the oscilloscope memory efficiently so the capture time, $T_{\text {capture }}$, is sufficient to achieve the required frequency resolution, $\Delta f=1 / T_{\text {capture }}$.

\section{Filtering and windowing}

Before acquiring the measurements, the appropriated record length must be selected in order to provide the required $\Delta f$. In that sense, the minimum capture time, $T_{m i n}$, and the minimum record length, $R L_{m i n}$, are given by,

$$
R L_{\min }=\operatorname{round}\left(f_{s} T_{\min }\right)=\operatorname{round}\left(f_{s} \frac{w_{f}}{\Delta f}\right),
$$

where, $w_{f}$ is the window factor of the windowing function to be applied in order to reduce the scallop loss and the spectral leakage caused by the finite length of the data.

After sampling and acquiring, the signal is digitally bandpass filtered to remove frequency components outside the band of interest, primarily noise measured below the minimum frequency of interest. The time delay introduced by the convolution operation between the filter's transfer function and the measured signal is then corrected.

The following step of the signal processing chain is windowing the data. The algorithm of this TDEMI measurement system use by default a Kaiser-Bessel window with the parameter $\beta=16.7\left(w_{f} \approx 2.23\right)$ since it offers a good compromise between amplitude accuracy and spectral leakage [15], which makes Kaiser-Bessel windows adequate to analyze both continuous and transient signals. However, the algorithm also offers the possibility of selecting other windowing functions such as the Gaussian, Hann, Hamming, Blackman or Flat-top that have been previously used in similar applications [14][16].

\section{Basic measurement algorithm}

As stated previously, our TDEMI measurement system is mainly intended to study transient, discontinuous and intermittent signals, still it must be able to handle continuous signals in order to provide a proper spectral estimation that includes narrow band and broadband signals, as required on the assessment of the radiated emissions.

Hence, the algorithm initially performs a preliminary measurement in order to identify transient, discontinuous and intermittent signals. This measurement must be configured for a capture time as long as possible, considering practical limitations such as memory restrictions. In our particular case, a record length, $R L$, of $10^{6}$ samples is used by default. As a general rule, $R L$ must be several times longer than $R L_{\text {min }}$ in order to provide an accurate spectral estimation. For example, for $f_{\max }=1 \mathrm{GHz}, \Delta f=120 \mathrm{kHz}$ and $w_{f}=2.23$, $R L_{\text {min }} \approx 9.3 \times 10^{4}$ samples, which is more than 10 times smaller than the selected $R L$.

Then, by manually adjusting manually the trigger to an appropriate level it is ensured to record at least a complete sample of the pulsed signal. Next, the pulse envelope and duration, $\Delta t_{\text {pulse }}$, are automatically and robustly calculated by the algorithm. It is recommended to set the oscilloscope to acquire in averaging mode since it helps obtaining a more consistent calculation of $\Delta t_{\text {pulse }}$, since it improves the signal-to-noise ratio by reducing the uncorrelated noise. The number of pulses, $n_{\text {pulses }}$, occurred during $T_{\text {capture }}$ is also counted. After analysing the preliminary measurement, one of the following different scenarios must be selected by the algorithm:

- If no pulse was detected, $n_{\text {pulses }}=0$, it is assumed that the measurement was triggered by a continuous signal, then the algorithm shall proceed with the spectral estimation using the preliminary measurement as input.

- If only one pulse was detected, $n_{\text {pulses }}=1$, it means the pulse repetition frequency is lower than de inverse of the capture time. Then, $R L$ is set so $T_{\text {capture }} \approx$ $\Delta t_{\text {pulse }}$ and the oscilloscope is configured to acquire in the FastFrame ${ }^{\mathrm{TM}}$ mode. The number of frames must be selected according the internal memory limitations. The accuracy in the estimation of the average pulse repetition frequency, $f_{\text {pulse }}$, increases with the number of frames. Through our experiments, we have found empirically that recording at least 10 pulse frames is sufficient to estimate consistently $f_{\text {pulse }}$, but that number of frames is dependent on the time base of the oscilloscope and may vary in other implementations.

- If a few pulses were detected (i.e., $2 \leq n_{\text {pulses }} \leq 10$, in our case), $f_{\text {pulse }}$ is estimated but it might not be a sufficiently accurate value. Therefore, in this scenario the oscilloscope is also configured to acquire in the FastFrame $^{\mathrm{TM}}$ mode for a capture time corresponding to the estimated pulse duration. Again, the number of frames is selected according the internal memory limitations, applying the same criteria explained above.

- If several pulses were detected $\left(n_{\text {pulses }}>10\right.$, in our case), it means the preliminary measure contains at least a pulsed signal with a $f_{\text {pulse }}$ properly estimable upon the initial measurement and then the algorithm shall proceed with the spectral estimation stage using the preliminary measurement as input.

\section{E. Equivalent "reassembled-time" signal}

If the algorithm decides to rerun the measures in FastFrame $^{\mathrm{TM}}$ mode, it measures several records of the pulsed signal accompanied by an absolute time stamp of the triggered events which occur in sequence. The time stamps are processed in order to provide a relative time of occurrence of the pulses and also to calculate $f_{\text {pulse }}$. It has been observed that the high resolution acquisition mode provides the best performance when measuring the pulses in the FastFrame ${ }^{\mathrm{TM}}$ mode since it allows obtaining better details of the measured pulse waveform by increasing the ADC resolution through oversampling and then providing a better dynamic range. No additional changes are required regarding the oscilloscope settings.

The individual pulses measured during each frame are merged in a equivalent "reassembled-time" signal using the information provided by the time stamps. Therefore, it is possible that the equivalent capture time exceeds the maximum record length allowable in a single run measure, while the effective memory usage remains manageable since this approach leads to a enormous data reduction. The time between pulses in the 
reassembled signal is, optionally, filled with the background noise of the initially recorded signal for the time lapse posterior the pulse has fallen and after the occurrence of the following pulse.

\section{F. Spectral estimation}

The developed TDEMI measurement system provides a few different Spectral Density Estimations (SDE) for the radiated emissions. For this purpose, the SDE is carried out by means of the Short-Time Fourier Transform (STFT) and the Welch's periodrogram. Therefore, the signal is examined in overlapped time windows of $T_{\min }$ duration in order to assure, by default, a minimum frequency resolution comparable to the $120 \mathrm{kHz}$ resolution bandwidth required by CISPR-16-1-1 for measurements in bands $\mathrm{C}$ and $\mathrm{D}$. However, the algorithm is able to adjust the window length to provide an reasonably arbitrary resolution bandwidth. The default overlapping percentage between windows is set to $75 \%$, being possible to increase it up to $90 \%$ (since a greater overlapping proportion would consume more memory without providing a significant improvement on accuracy) or decrease it down to $50 \%$ (when reducing the computation cost is needed) in order to adjust resolution in time domain.

The STFT is used to analyze the spectral content of local sections of a measured signal as it changes over time. The STFT provides a spectrogram matrix from which is possible to provide a worst case spectral estimation comparable with a measurement obtained with a max-peak detector. On the other hand, Welch's periodogram combines the STFT method with the averaging of each modified periodograms of the windowed time frames, providing a SDE comparable with a measurement obtained with an average detector. For a detailed explanation of spectral estimation techniques, please consult [17].

\section{G. Correction and scaling of measurements}

Since each component of the system (antenna, cables, preamplifier, oscilloscope) has a specific impulse response, it would be required to correct the direct oscilloscope voltage measurements, $v(t)$, in order to obtain the corresponding electric field as it varies over time, $E(t)$. However, this would depend upon knowing or measuring the impulse response of all the elements of the measurement chain and then deconvolve it of the measured signal. This might be neither possible nor practical for most EMC measurements and, therefore, it result more convenient to apply corrections in the frequency domain.

Assuming that only the amplitude spectrum representation is required, our TDEMI measurement system applies the corresponding correction factors such as cable attenuation and signal path losses, antenna factors and the oscilloscope frequency response. Since the EMI spectrum is meant to be expressed in terms of decibels-microvolts per meter, the aforementioned correction factors are added, as usual, and the adequate scaling factors are incorporated for assuring consistency in the units.

\section{H. Budget}

In general terms, the oscilloscope is the most expensive equipment involved in the whole setup of the implemented TDEMI measurement system. For measurements up to $1 \mathrm{GHz}$, very affordable and versatile hardware options are available in the market including USB PC-based scopes which are relatively inexpensive (about $€ 5,000$ ) and very practical for in-situ measurements due to its size and weight. For measurements above $1 \mathrm{GHz}$, similar alternatives are also available for higher budgets being relatively common to found suitable oscilloscopes with a measuring bandwidth of $10 \mathrm{GHz}$ and more for less than $€ 20,000$. In the near future, it's expected those prices will be reduced. Regarding the rest of the hardware, a quick survey have allowed us to estimate that an amount of $€ 1,500$ extra would be sufficient to procure the required accessories and personal computer. Nevertheless, in most cases, elements such as antennas, cables and preamplifiers are interchangeable items that are already available in an EMC lab, being unnecessary to include them in the overall cost of the TDEMI measurement system.

It is important to state that we have intentionally not mentioned references for the aforementioned prices because it is not our intention to advertise any vendor, however, this information can be easily verified by anyone who is interested.

\section{Notes on the software implementation}

All the signal processing algorithms applied after the PC acquisition stage are implemented in MATLAB ${ }^{\circledR}$ due its conveniently broad library of mathematical functions and its optimized approach for operations involving matrices and vectors. Statistics Toolbox and Signal Processing Toolbox functions are used throughout the code.

\section{VAlidation Methodology}

The validation of the presented TDEMI measurement system was performed for radiated emissions testing by means of well-known controlled transient and continuous signals generated using a burst generator Schlöder SFT 1400, an arbitrary signal generator Agilent $81160 \mathrm{~A}$ and a programmable synthesizer Hameg HM8134 as the source of the EMI. The EMI was radiated by an open cable and/or by antennas, respectively. The measurements were performed inside an anechoic chamber. Regarding the measuring antenna, a BiLog antenna Schaffner CBL6143 was used. The results were compared with those obtained from a conventional EMI test receiver model ESPI from Rohde\&Schwarz. A general diagram of the measurement setup is shown in Figure 3.

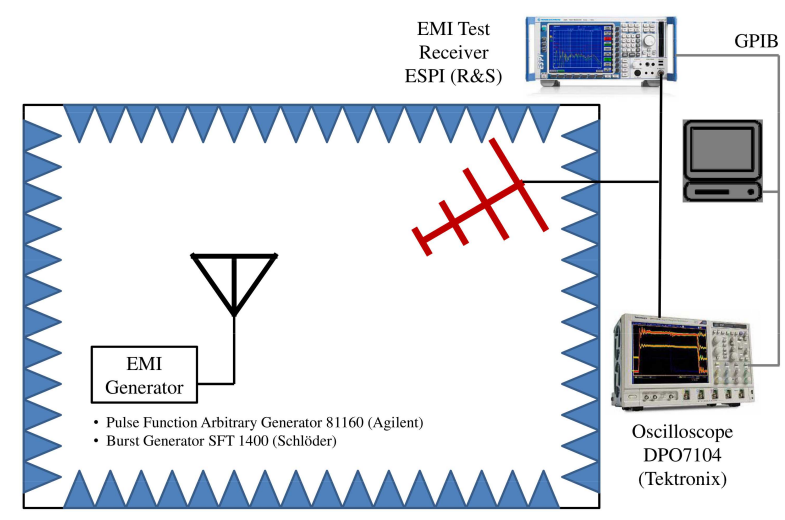

Fig. 3. Simplified experimental setup for the validation of the TDEMI measurement system. 
Since for transient signals with a low pulse repetition frequency a single sweep measurement of the EMI test receiver is likely to provide an incorrect result for the spectral content of the disturbance, several sweeps of the EMI test receiver were required with the max hold enabled until the measurement was useful for comparison purposes. Finally, the Feature Selective Validation (FSV), a method widely known in the field of computational electromagnetics [18], was used for assessing a comparison between the measurement results obtained with the developed system with respect to measurements taken with the conventional EMI test receiver.

\section{RESUlts}

The following subsections present a pair of representative measurement results for which our TDEMI system has been validated. However, several other controlled validation stages has been performed previously in order to assure the correctness and consistency of our measurements procedures, algorithms, instruments and reference patterns.

\section{A. Radiated burst}

In this experiment, a radiated burst is measured as a representative case of a broadband EMI formed by single periodic pulses with $f_{\text {pulse }} \ll B_{\text {imp }}$ (impulse bandwidth). Particularly, our burst generator was set at $5 \mathrm{kHz}$ with a $15 \mathrm{~ms}$ pulse duration and a burst period of $50 \mathrm{~ms}$. Let us assume we are only interested in measuring the spectral components corresponding the aforementioned pulsed EMI. Since the standard burst (IEC 61000-4-4) has a rise time of $5 \mathrm{~ns}$, it is a priori known that its radiated emissions are entirely expected in the $30 \mathrm{MHz}-300 \mathrm{MHz}$ band, therefore $f_{s}=1.25 \mathrm{GHz}$ is used. Antialiasing filtering was not required. The TDEMI and the corresponding ESPI test receiver were configured for measuring using coherent parameters: $\Delta f=120 \mathrm{kHz}$, Max Peak and Average detector. The ESPI Test receiver was configured to capture 8000 frequency points (maximum) with the max-hold feature active in order to capture the worst case emissions after several sweeps of $5 \mathrm{~s}$ duration each. The results corresponding to the EMI spectral estimation are shown in Figure 4 for both detectors and both measurement systems.

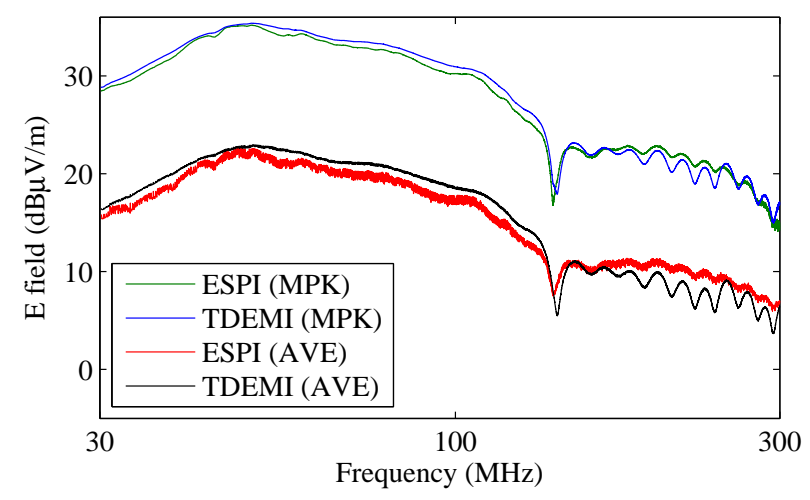

Fig. 4. Spectral estimation for a radiated burst pulse.

The difference between both measuring methods was of less than $0.4 \mathrm{~dB}$ at the frequency of maximum emission level. Then, applying the FSV, the overall results indicate that there is, in average for all the detectors, an "Excellent"-"Very Good" level of agreement between the traces regarding their amplitude $\left(A D M_{t o t}=0.114\right)$, feature $\left(F D M_{t o t}=0.2724\right)$ and global $\left(G D M_{t o t}=0.3177\right)$ difference measures. However, the validation results might have been degraded by the noise in the traces.

\section{B. Superposition of Broadband and Narrowband EMI}

Our second experimental scenario deals with an EMI formed by a periodic transient pulse of $f_{\text {pulse }}=100 \mathrm{kHz}$ and a continuous tone at $900 \mathrm{MHz}$. Now, let's assume it is required measuring the whole spectrum covering form $30 \mathrm{MHz}$ up to $1 \mathrm{GHz}$, thus setting $f_{s}=5 \mathrm{GHz}$. Again, the TDEMI and the corresponding ESPI test receiver were configured for measuring using coherent parameters: $\Delta f=120 \mathrm{kHz}$, Max Peak and Average detector and the maximum number of samples corresponding in each case. The results corresponding to the EMI spectral estimation are shown in Figure 5 for both detectors and both measurement systems.

In Figure 5, the SDE provided by our TDEMI measurement system was performed using the reassembled signal constructed by superposing, in the time domain, the transient and continuous parts. The results indicate that our TDEMI measurement system provides results within $\pm 1.5 \mathrm{~dB}$ when compared with a conventional EMI test receiver, provided that the signal level is above the noise floor of both measurement systems. Considering that, typically, the radiated emissions measurements have an expanded uncertainty higher that $3 \mathrm{~dB}$, our system provides a comparable accuracy level.

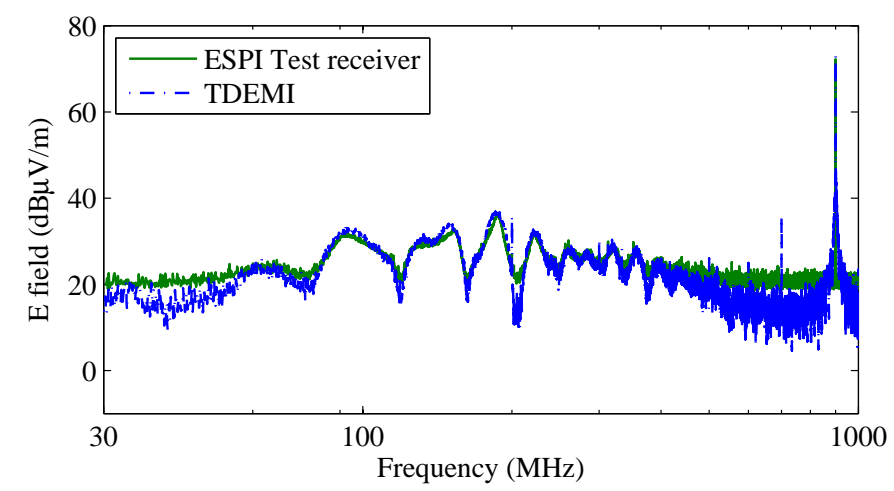

(a) Max-Peak detector

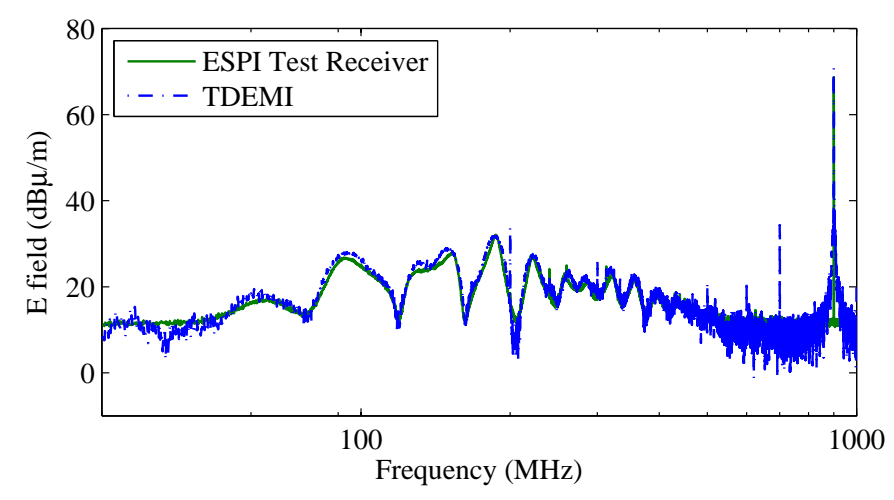

(b) Average detector

Fig. 5. EMI spectral estimation superposed broadband and narrowband EMI. 
In this particular case, the standard FSV indicates that the overall level of agreement between the ESPI Test Receiver and our TDEMI measurement system results is, in average for all the detectors and methods, "Good"-"Fair" for their amplitude $\left(A D M_{t o t}=0.5463\right)$, feature $\left(F D M_{t o t}=0.6882\right)$ and global $\left(G D M_{t o t}=0.9832\right)$ difference measures. However, careful must be take when comparing measurement results applying the standardized FSV because if such measurement include noisy frequency bands, as this particular case, the overall indicators provide misleading results and they should be accompanied by a proper uncertainty statement for the validation results [19].

\section{CONCLUSION}

A TDEMI measurement system, that employs an alternative approach and different algorithms in comparison to previously published works that addresses this subject, has been presented. One of the principal advantages of our TDEMI system is that it can be continuously improved through signal processing implemented via software. Even if our general purpose equipment limits the measurement band up to $1 \mathrm{GHz}$, it would be completely feasible to enhance the current capabilities of the TDEMI measurement system by using an oscilloscope with a larger bandwidth.

As expected, the measuring and processing time required by our TDEMI measurement system is much lower than the required by conventional EMI test receivers, however, we believe this is not the most relevant improvement achieved. In our TDEMI measurement system the algorithms detect robustly the transient EMI pulses and adjust automatically the oscilloscope parameters and acquisition modes to capture only the pulse of interest with the optimal instrument configuration, thus the measuring system manage more effectively aspects such as the dynamic range and the ambient noise. Implicitly, those features also reduces the amount of samples required for a proper SDE of the measured EMI, and therefore, it provides an improved capability for managing the required oscilloscope memory, even if this aspect was not discussed in detail in the main body of the paper.

On the other hand, one remarkable feature of our approach to the TDEMI measurement system implementation is that it was feasible to built it with a relatively small budget, starting approximately form $€ 5,000$; proving that radiated EMI assessment by means of time domain techniques is not only very accurate but also affordable for small in-company or university EMC laboratories which require a fast and cost effective alternative to evaluate accurately the performance of the radiated emissions of their products and prototypes.

The authors believe that TDEMI systems, as the one presented in this article, are an attractive alternative for performing in-situ measurements on industrial environments or under situations where the equipment under test can't be properly installed within a controlled measurement facility such a semianechoic chamber, since it would reduce significantly the cost of performing or contracting such measurements.

\section{ACKNOWLEDGMENT}

This work was supported in part by EURAMET IND60EMC research project (the EMRP is jointly funded by the EMRP participating countries within EURAMET and the European Union) and by the Spanish "Ministerio de Economía y Competitividad", under project TEC2013-48414-C3-3-R.

\section{REFERENCES}

[1] P. Torio and M. Sanchez, "Novel Procedure to Determine Statistical Functions of Impulsive Noise," Electromagnetic Compatibility, IEEE Transactions on, vol. 47, no. 3, pp. 559-568, Aug 2005.

[2] R. Adriano, N. Ben Slimen, V. Deniau, M. Berbineau, and P. Massy, "Prediction of the BER on the GSM-R communications provided by the EM transient disturbances in the railway environment," in Electromagnetic Compatibility - EMC Europe, 2008 International Symposium on, Sept 2008, pp. 1-5.

[3] M. Pous and F. Silva, "Full-Spectrum APD Measurement of Transient Interferences in Time Domain," Electromagnetic Compatibility, IEEE Transactions on, vol. PP, no. 99, pp. 1-9, 2014.

[4] _ - "Prediction of the Impact of Transient Disturbances in Real-Time Digital Wireless Communication Systems," Electromagnetic Compatibility Magazine, IEEE, vol. 3, no. 3, pp. 76-83, rd 2014.

[5] H. Westenberger, "Use of Time Domain methods for CISPR16 compliant EMI Measurements," in Microwaves, Communications, Antennas and Electronics Systems, 2009. COMCAS 2009. IEEE International Conference on, Nov 2009, pp. 1-4.

[6] P. Russer, "EMC measurements in the time-domain," in General Assembly and Scientific Symposium, 2011 XXXth URSI, 2011, pp. 1-35.

[7] Specification for radio disturbance and immunity measuring apparatus and methods - Part 1-1: Radio disturbance and immunity measuring apparatus - Measuring apparatus, CISPR Std., july 2010.

[8] G. Costa, M. Pous, A. Atienza, and F. Silva, "Time-Domain Electromagnetic Interference Measurement System for intermittent disturbances," in Electromagnetic Compatibility (EMC Europe), 2014 International Symposium on, Sept 2014, pp. 833-837.

[9] S. Braun and P. Russer, "Requirements and Solutions for Emission Measurements in Time-Domain According to International EMC Standards," in Electromagnetic Compatibility (APEMC), 2012 Asia-Pacific Symposium on, May 2012, pp. 209-212.

[10] F. Krug, D. Mueller, and P. Russer, "Signal Processing Strategies with the TDEMI Measurement System," Instrumentation and Measurement, IEEE Transactions on, vol. 53, no. 5, pp. 1402-1408, Oct 2004.

[11] F. Krug and P. Russer, "Quasi-peak Detector model for a TimeDomain Measurement System," Electromagnetic Compatibility, IEEE Transactions on, vol. 47, no. 2, pp. 320-326, May 2005.

[12] C. Keller and K. Feser, "Non-Linear Superposition Of Broadband Spectra For Fast Emission Measurements In Time Domain," in Proceedings of 15th Zurich EMC Symposium, 2003, pp. 505-510.

[13] C. Hoffmann and P. Russer, "A Time-Domain system for EMI measurements above $1 \mathrm{GHz}$ with high sensitivity," in Microwave Conference (GeMIC), 2011 German, March 2011, pp. 1-4.

[14] C. Keller and K. Feser, "Fast Emission Measurement in Time Domain," Electromagnetic Compatibility, IEEE Transactions on, vol. 49, no. 4, pp. 816-824, Nov 2007.

[15] J. Solomon, O., "The Use of DFT windows in Signal-to-Noise ratio and Harmonic Distortion Computations," Instrumentation and Measurement, IEEE Transactions on, vol. 43, no. 2, pp. 194-199, Apr 1994.

[16] M. Keller, "Comparison of Time Domain Scans and Stepped Frequency Scans in EMI Test Receivers," Rohde \& Schwarz White Paper, 122013.

[17] D. Manolakis and V. Ingle, Statistical and Adaptive Signal Processing: Spectral Estimation, Signal Modeling, Adaptive Filtering, and Array, ser. Communications and signal processing. McGraw-Hill, 2011.

[18] A. Duffy, A. Martin, A. Orlandi, G. Antonini, T. Benson, and M. S. Woolfson, "Feature Selective Xalidation (FSV) for Validation of Computational Electromagnetics (CEM). Part I-the FSV method," Electromagnetic Compatibility, IEEE Transactions on, vol. 48, no. 3, pp. 449459, Aug 2006.

[19] M. Azpurua, E. Paez, J. Rojas-Mora, O. Ventosa, F. Silva, G. Zhang, A. Duffy, and R. Jauregui, "A Review on the Drawbacks and Enhancement Opportunities of the Feature Selective Validation," Electromagnetic Compatibility, IEEE Transactions on, vol. 56, no. 4, pp. 800-807, Aug 2014. 